\title{
Predicting the Port-catch Volume of Salmon at Eastern Hokkaido
}

\author{
Yue Zhang ${ }^{1}$, Hiroyuki Shioya ${ }^{1}$, and Masaaki Wada ${ }^{2}$ \\ ${ }^{1}$ Muroran Institute of Technology, 27-1mizumoto-Cho, Muroran, Hokkaido, Japan \\ ${ }^{2}$ Future University Hakadate, 116-2 Kamedanakano-cho, Hakodate, Hokkaido, Japan
}

Keywords: estimations, LSTM, extended data, Fishery Volume

As we all know, freshness determines the value of aquatic products. The shorter the interval between the start of fishing and the sale, the greater the profit (Nicolae et al. 2015). Especially in the vast Hokkaido area, it is very necessary to make an effective and scientific transportation plan. In this process, the estimation of the port capture can greatly help the overall planning of the transportation system and improve transportation efficiency.

Deep learning methods are widely used in a variety of fields, including aquaculture (Benzer and Benzer 2015), fish identification (Monkman et al. 2019), and fishery catch prediction (Shiichi and Teruhisa 2000). Because of its ability to mine long-distance time series data, the Long Short-Term Memory Network (LSTM) (Hochreiter and Schmidhuber 1997), a machine learning method based on recurrent neural networks, has been widely employed in speech recognition, machine translation, data prediction, and other domains. Therefore, we use this method to forecast the port catch.

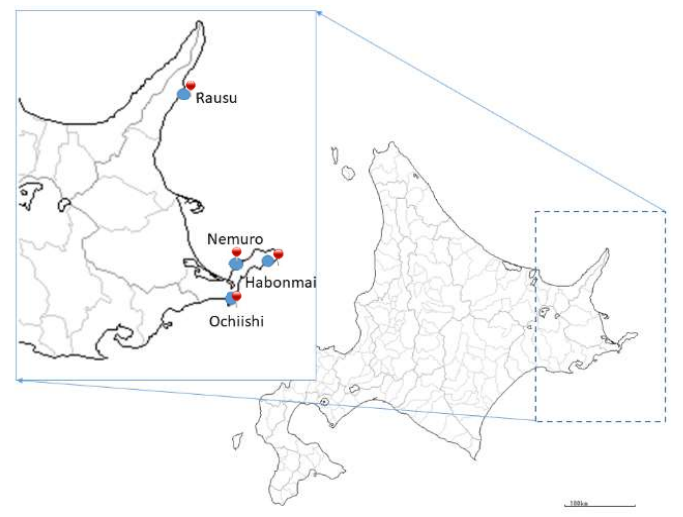

Fig. 1. Position of ports in eastern Hokkaido.

This experiment used the fishery catch in four ports (Nemuro, Ochiishi, Habomai, and Rausu), from 1 June 2005 to 30 November 2015 (Fig. 1). For water temperature data, we used satellite data from the NEAR-GOOS website and the same time interval (the temperature of $50 \mathrm{~m}$ ). The data of the first 20 days beginning 1 June were used to predict the catch value for the next day.

In the experiment section, we propose a data enhancement method to address the issues of insufficient data and over-fitting of the network model. The model processes both the original and the added data, and the experimental results are compared.

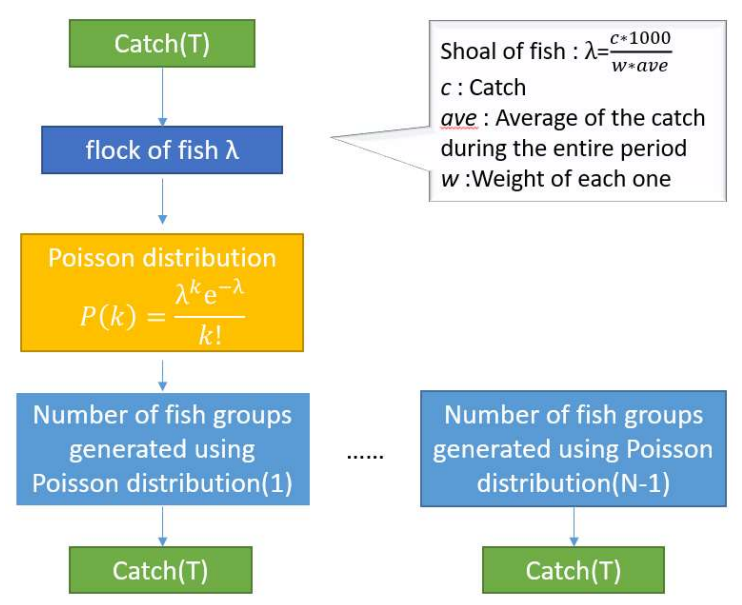

Fig. 2. Extended data based on Poisson distribution.

All correspondence should be addressed to Y. Zhang. 
Since Poisson distribution corresponds to discrete natural phenomena, it is often used in fishery resource analysis. Considering the problem of fish arriving at the fishing range of a port, this can be seen as a queuing problem, and thus the Poisson distribution can model this phenomenon very well.

To expand the training set, we used the Poisson model to generate extended data in the training set. We extracted data on port-catch volume in the training set, then assumed a fish had a weight of $w=3 \mathrm{~kg}$ and treat $\mathrm{K}$ as the number of fish in one group of fish. To begin, the port-catch volume (tonnes) was converted into the number of shoals of fish, and the shoal data was fed into the Poisson distribution to generate extended data. Finally, the data were restored to tonnes (Fig. 2). The reason for changing the tonnage of the fish to shoals of fish is twofold: first, increasing the $\lambda$ of Poisson distribution will reduce the fluctuation of the Poisson distribution, thereby reducing the error and bringing the artificial data closer to the real data. Second, this method of data processing mimics the natural motion of fish.

Regarding the setting of the coefficient $N$ (the number of fish in each group), since the data scale of each fishing port is different, the size of the fish school is also different. Therefore, we calculated the average value of the catch data of each fishing port as a benchmark for $K$ value and observed the effect of the change in $K$ value on the experimental error to find the most suitable $K$ value.

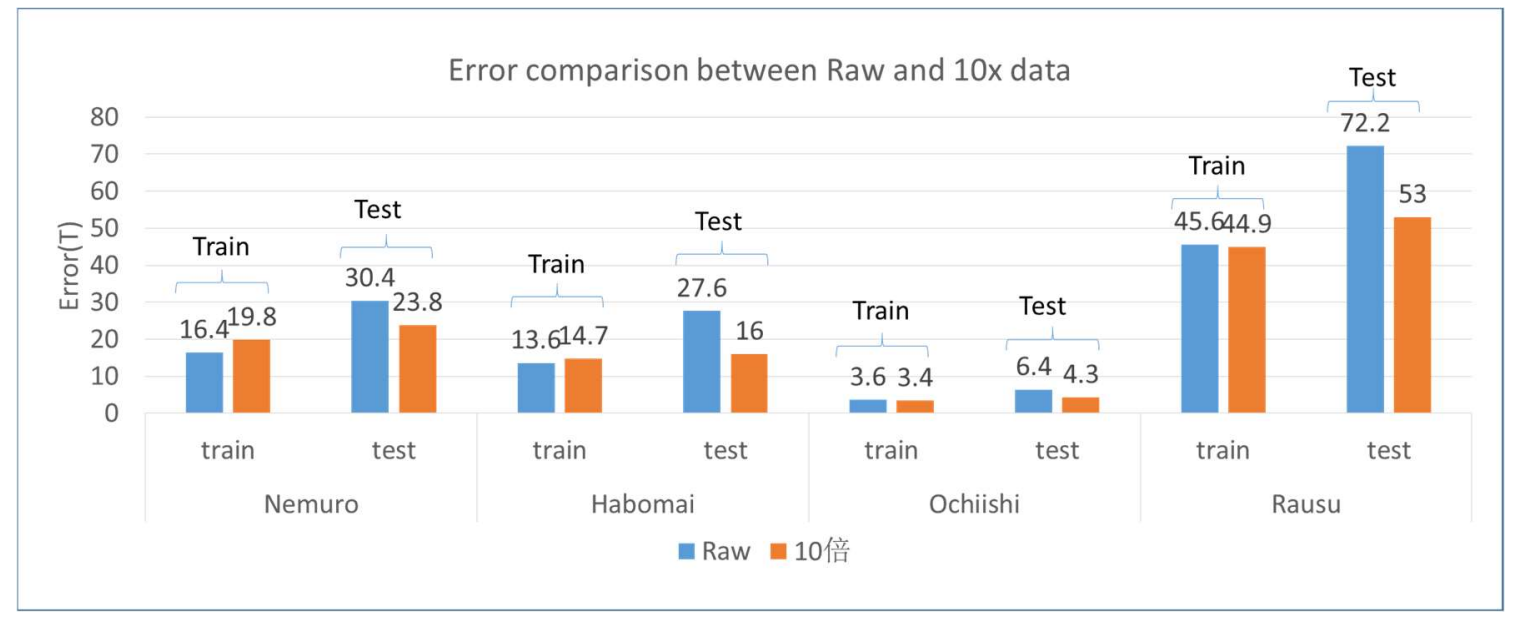

Fig. 3. Error of two situations.

According to the experimental results, we used the Root Mean Square Error (RMSE) to evaluate the experimental results, comparing the RMSE in different models and different training sets (Fig. 3).

For the experimental results, we can see that it is feasible to use the LSTM model in RNN to predict fishery catch data. Some processing methods for the original data also have a certain effect. By increasing the training set through experimental methods, the test error of the model can be increased to a certain extent, and the problem of model over-fitting on the original data set can be alleviated.

This research will help the use and maintenance of aquatic resources. Through the prediction of data and the efforts made to reduce the prediction error, we can use the forecast of fishing port capture to guide and support fishery logistics and transportation.

\section{REFERENCES}

Benzer, R., and S. Benzer. 2015. Application of artificial neural network into the freshwater fish caught in Turkey. Int. J. Fish. Aquat. Stud. 2(5): 341-346.

Hochreiter, S., and J. Schmidhuber. 1997. Long short-term memory. Neural Comput. 9(8): 1735-1780.Nicolae, C.G., L.M. Moga, M.I. Nenciu, G.V. Bahaciu, and M.P. Marin. 2015. Particularities and management of the distribution chain for fish and fishery products. AgroLife Sci. J. 4(1): 111-116.

Monkman, G.G., K. Hyder, M.J. Kaiser, and F.P. Vidal. 2019. Using machine vision to estimate fish length from images using regional convolutional neural networks. Methods Ecol. Evol. 10(12): 2045-2056.

Shoichi, I., and K. Teruhisa. 2000. Forecasting for the catch of yellowtail larva by a neural network. Fisheries biology and oceanography in the Kuroshio. pp. 39-43. (In Japanese) 